\title{
Macromodelos odontológicos impressos em 3D como recurso complementar ao ensino em escultura dentária
}

\author{
Andreia de Oliveira Souza*; Daiane Cristina Peruzzo**; Fabiana Mantovani Gomes França**; Arlete \\ Maria Gomes de Oliveira**; Kamilla Rosamilia Kantovitz**
}

* Mestranda em Clínica Integrada, Faculdade São Leopoldo Mandic
** Professora Doutora, Faculdade São Leopoldo Mandic

Recebido em 28/01/2018. Aprovado em 18/11/2018.

\begin{abstract}
RESUMO
A diversificação das práticas pedagógicas visa o aprimoramento do processo de ensino. Novas metodologias, que favoreçam o interesse dos alunos e que proporcionem melhor compreensão, podem ser ferramentas importantes no processo ensino-aprendizagem. O objetivo desse estudo foi avaliar a percepção dos estudantes sobre o uso de macromodelos odontológicos, confeccionados com impressora 3D, como recurso complementar de ensino na disciplina Escultura Dentária, no curso de graduação em Odontologia da FACIPLAC. A amostra foi composta por 44 estudantes, matriculados no quinto período. Foram produzidos 44 conjuntos com quatro macromodelos, sendo um incisivo central, um canino, um primeiro pré-molar e um primeiro molar, todos superiores, confeccionados em material plástico colorido e resistente. Os conjuntos foram entregues aos estudantes no início de cada aula e utilizados para demonstração das estruturas anatômicas, visando à melhor fixação do conhecimento, sendo recolhidos posteriormente. Ao final do semestre letivo foi aplicado um questionário relativo ao aproveitamento do uso dos macromodelos, com resposta de 38 estudantes. Os resultados indicam que o uso dos macromodelos foi válido como recurso facilitador da compreensão da técnica de escultura dentária; contribuindo com o processo de aprendizagem em comparação ao uso de diapositivos; destacando as dificuldades no aprendizado; auxiliando na sedimentação dos conceitos das formas geométricas das estruturas dentárias. Os estudantes indicam seu uso, contudo consideram que não substitui a demonstração prática pelo docente.
\end{abstract}

Descritores: Escultura. Ensino. Aprendizagem. Impressão Tridimensional. Anatomia.

\section{INTRODUÇÃO}

Atualmente, caminhos inovadores têm sido apontados para a capacitação, formação e motivação dos acadêmicos de Odontologia.
Formas diferenciadas de ensino e aprendizagem têm sido adotadas na perspectiva de interligar a teoria com a prática laboratorial e clínica, além de desenvolver ações originais e criativas 
capazes de modificar o resultado final do aprendizado $^{1}$.

O método de ensino tradicional baseado na aula com giz, lousa e apostilas há tempo foi substituído por aulas expositivas construídas em slides e softwares. A necessidade de reformular constantemente a educação tem sido proposta levando em consideração as novas exigências colocadas pelos meios de comunicação social, no que diz respeito ao conteúdo e também em relação à forma ${ }^{2}$.

Métodos de ensino tradicionais e ultrapassados não favorecem a criatividade e a inteligência dos jovens. Além disso, a eficiência da aprendizagem nas universidades é muito baixa quando são utilizados os métodos clássicos. Logo, é preciso modernizar a educação para acompanhar as transformações tecnológicas ocorridas no mundo ${ }^{3}$.

A impressão tridimensional surgiu em 1984, inventada por Chuck Hull, um norteamericano do estado da Califórnia. Também conhecida como prototipagem rápida, é uma forma de fabricação aditiva, onde um modelo tridimensional, obtido a partir de um projeto digital é criado por sucessivas camadas de material. Estes modelos podem ser criados por meio de softwares de modelagem tridimensional ou de digitalização em três dimensões ${ }^{4}$. Os materiais de construção mais utilizados atualmente são poliácido lático (PLA) e acrilonitrila butadieno estireno (ABS). Tais materiais plásticos têm boas propriedades mecânicas e podem ter aplicação em diversas áreas da indústria e no uso doméstico ${ }^{5,6}$.

Assim, a globalização e a informatização da sociedade requerem a modernização dos métodos e dos recursos no processo de ensino e aprendizagem. O grande desafio pedagógico, diante das inovações contemporâneas, é identificar como os professores, detentores de notório conhecimento técnico-científico e de reconhecida especialização e mérito em sua área de atuação, utilizariam as novas tecnologias em suas práticas pedagógicas na construção de um ensino de melhor qualidade. Uma das possibilidades é empregar a tecnologia como auxiliar no processo de ensino ativo, inteligente, com linguagem de fácil interpretação, com o intuito de facilitar a compreensão e a fixação do conhecimento $^{7-9}$.

Os estudos envolvendo macromodelos confeccionados com impressora 3D como auxiliar no processo de ensino-aprendizagem ainda são escassos na literatura. Neste contexto, esta pesquisa buscou avaliar a utilização de macromodelos odontológicos, confeccionados em impressora 3D, no aprendizado de escultura dentária, bem como analisar a percepção do estudante de graduação ao utilizá-los, verificando sua influência na sedimentação dos conceitos e das formas geométricas das estruturas dentárias, com seu real arranjo espacial.

\section{MATERIAL E MÉTODOS}

Este estudo foi conduzido de acordo com os preceitos determinados pela Resolução 466/12, do Conselho Nacional de Saúde do Ministério da Saúde, e submetido à apreciação do Comitê de Ética em Pesquisa da Faculdade São Leopoldo Mandic, recebendo aprovação, com parecer número 2.115.848.

A amostra foi de 44 estudantes do quinto semestre do curso de graduação em Odontologia das Faculdades Integradas da União Educacional do Planalto Central (FACIPLAC), em BrasíliaDF, matriculados na Disciplina de Escultura Dentária.

Para a construção dos macromodelos odontológicos, foram utilizados filamentos de ABS de 1,75 mm de diâmetro, um material resistente, manipulável, leve e lavável, nas cores rosa, amarela, azul e verde (figura 1). 


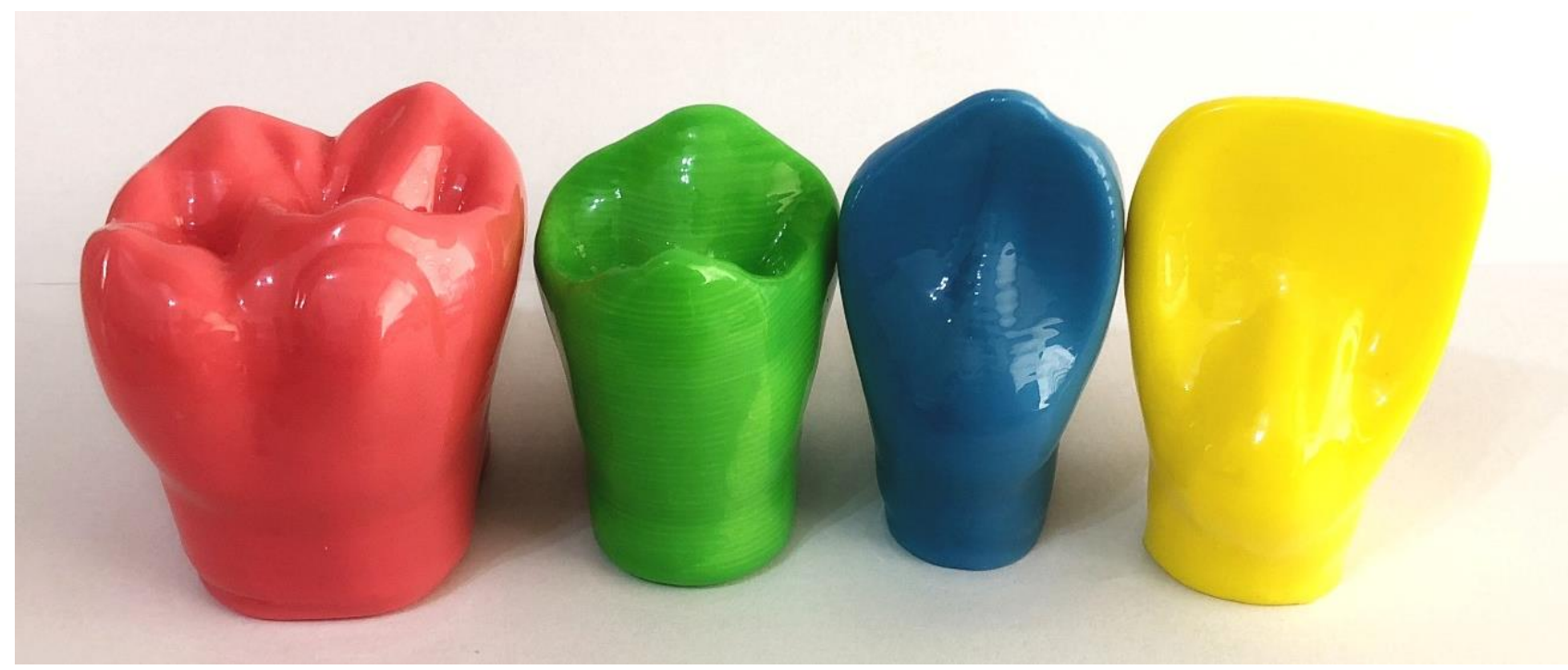

Figura 1. Macromodelos utilizados.

Foram confeccionados kits com quatro macromodelos tridimensionais, de aproximadamente 6,5 centímetros de altura, sendo um incisivo central, um canino, um primeiro prémolar e um primeiro molar, todos superiores, do lado direito, coloridos aleatoriamente, que foram entregues a cada um dos estudantes. Tais macromodelos foram utilizados para demonstração das estruturas anatômicas dentárias, tais como cíngulo, cúspide, face, aresta, vertente, crista, sulco, fossa, zênite, tubérculo e linha do colo. Todos eles foram recolhidos ao final das atividades. Apresentações de slides e livros não foram utilizados.

Os estudantes poderiam comparar o método utilizado, com uso de macromodelos impressos em 3D, apenas com o método tradicional utilizado em outras disciplinas, em que somente são utilizados livros e apresentações de diapositivos.

Terminado o semestre letivo, foi aplicado um questionário validado e adaptado, composto por seis questões fechadas (quadro 1), além de uma questão aberta destinada a comentários e sugestões. A participação dos alunos foi voluntária, após assinatura de termo de consentimento livre e esclarecido (TCLE).

Os dados foram tabulados e são apresentados na forma de frequências absoluta e relativa referente a cada pergunta. A análise dos dados serviu para interpretar as percepções e opiniões a respeito de novos métodos aplicados sobre o processo de ensino e aprendizagem. Os comentários e sugestões foram compilados e apresentados nos resultados.

\section{RESULTADOS}

Dos 44 estudantes matriculados na disciplina de Escultura Dentária que receberam o questionário, $39 \quad(88,63 \%) \quad$ o devolveram respondido, contudo um deles não assinou o TCLE, resultando em taxa de resposta de 86,36\%.

A média de idade dos participantes foi de 25 anos, variando entre 19 e 47, com desvio-padrão de 5,87 . Responderam pertencer ao gênero masculino 11 estudantes $(28,95 \%)$ e feminino 27 (71,05\%). As respostas são apresentadas no gráfico 1 .

No campo destinado a comentários e sugestões, 7,89\% dos respondentes relataram que os macromodelos 3D "são uma ótima ferramenta para auxiliar nas aulas práticas de escultura, principalmente quanto às estruturas anatômicas"; 
"facilitam a visualização das estruturas que temos dificuldades de visualizar nos slides"; e "além de serem lindos e muito bem confeccionados, ajudam na fixação de cada estrutura dentária".

\section{Quadro 1. Questionário.}

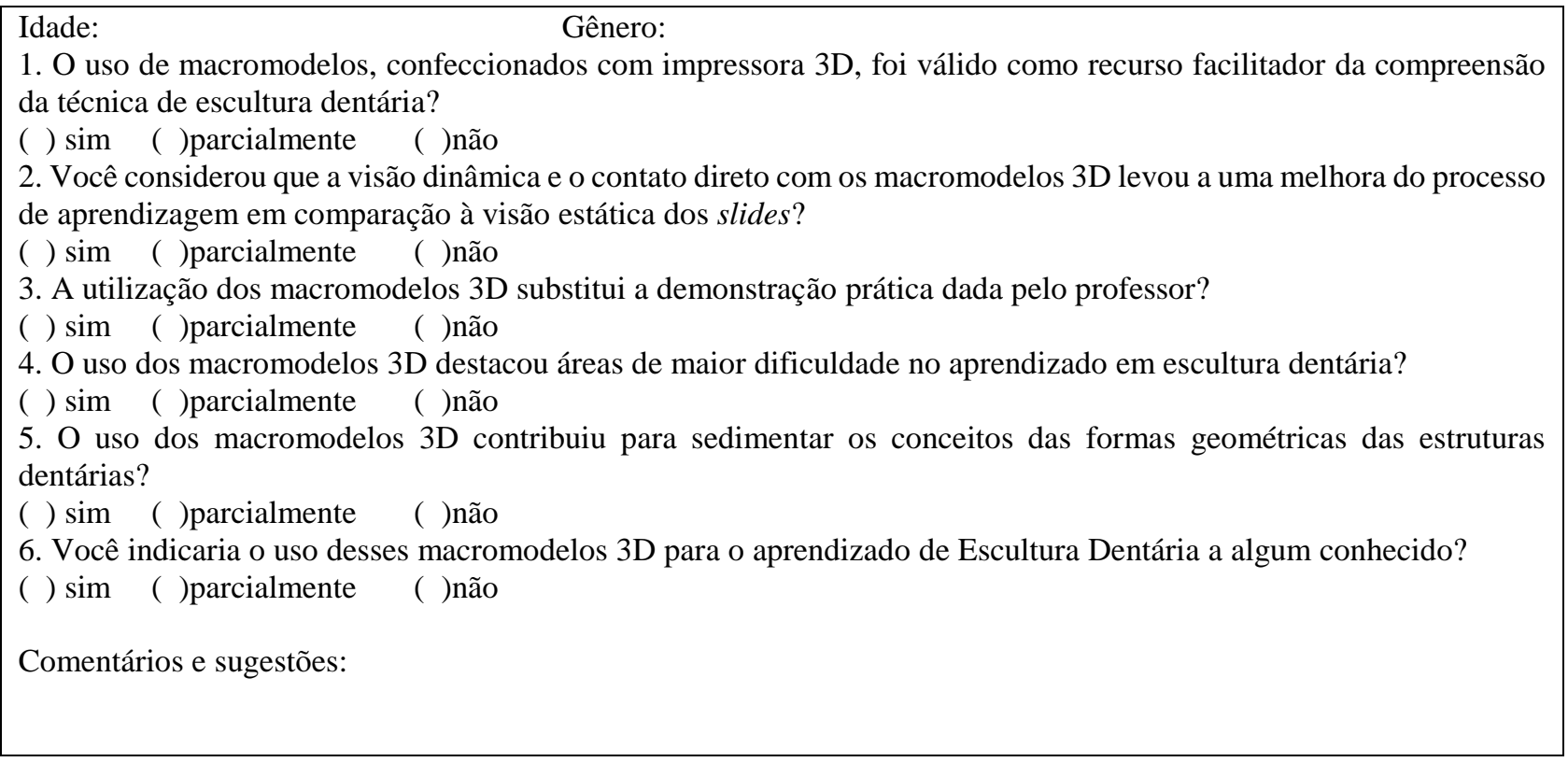

Gráfico 1. Respostas dadas às perguntas fechadas do questionário.

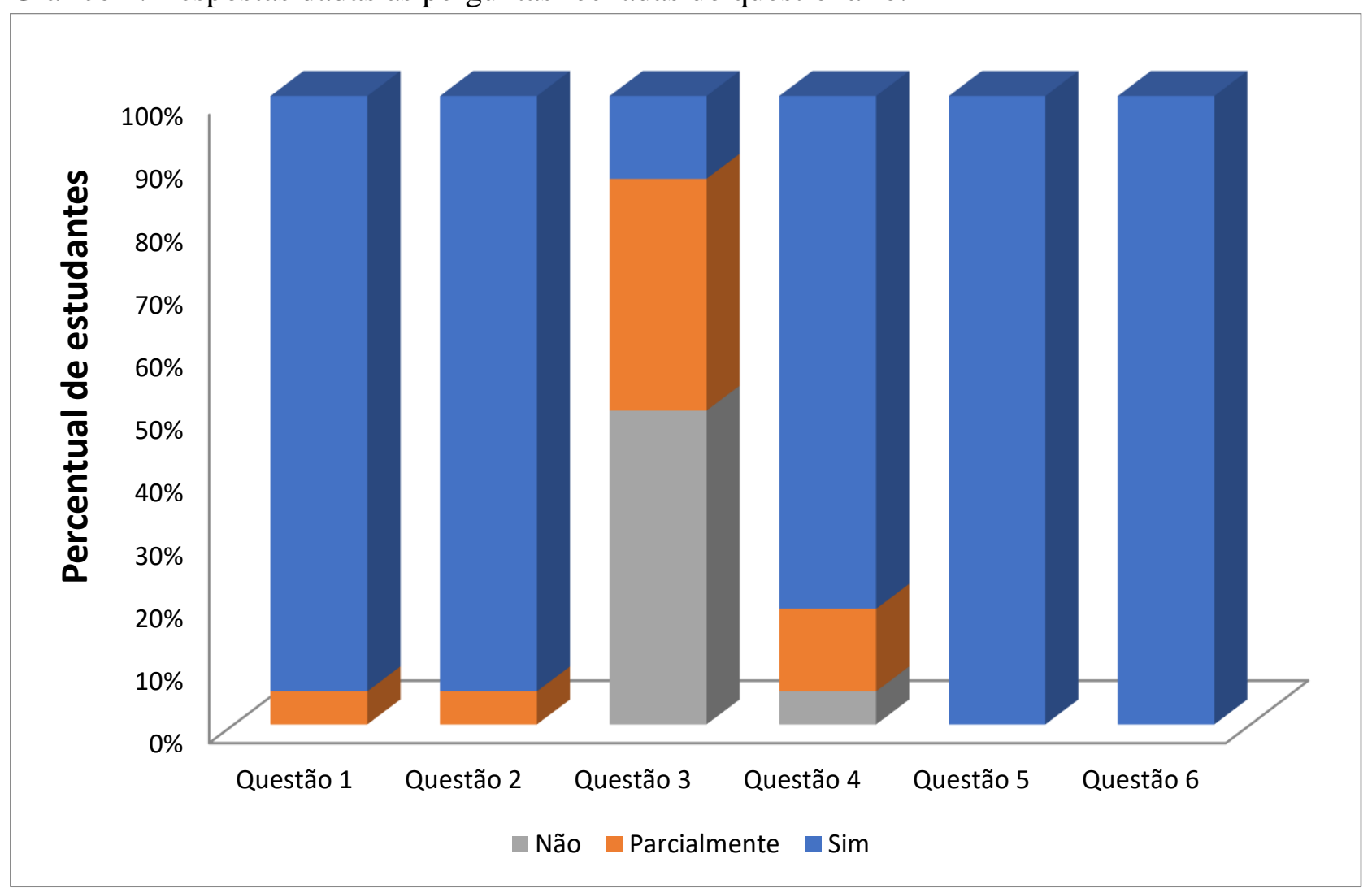




\section{DISCUSSÃO}

A absoluta maioria dos estudantes respondeu que o uso dos macromodelos foi válido como recurso facilitador da compreensão da técnica de escultura dentária e que melhorou o processo de aprendizagem a partir da visão dinâmica e do contato direto, quando comparado à visão estática dos slides. Em uma disciplina essencialmente prática, qualquer recurso auxiliar que possa despertar os sentidos dos estudantes poderia ser capaz de provocar tal resposta. Contudo, a ausência de qualquer resposta negativa evidenciou qualidades e deu aos macromodelos tridimensionais uma importância significativa na construção do conhecimento dos estudantes.

A percepção tridimensional se desenvolve à medida em que um indivíduo vivencia o espaço, principalmente por meio da visão e do tato, que captam estímulos, como brilho, sombra, cor, tipo de contato, entre outros. Esses estímulos vão para o cérebro, que os interpreta elaborando conceitos de forma, proporção, posição e orientação. Assim, todo esse processamento leva à formulação de novos conceitos ou ao refinamento dos já existentes ${ }^{10}$. Portanto, é natural que os macromodelos odontológicos feitos com impressora 3D sejam realmente um recurso facilitador da compreensão da técnica de escultura dentária.

Na mesma linha, mais de $80 \%$ dos estudantes destacaram áreas de maior dificuldade no aprendizado em escultura dentária com o uso dos macromodelos 3D. A memória tem papel fundamental na inteligência espacial. $\mathrm{O}$ raciocínio espacial se refere à manipulação imaginativa de entes espaciais. Depois da imagem do modelo ter sido formada na mente, esta manipulação é feita com certa facilidade. Normalmente, ao apreciar-se um objeto, instintivamente se pega nele, girando-o e observando-o em vários ângulos. Nesse procedimento, utilizam-se os sentidos da visão e do tato. Assim, percebem-se detalhes do objeto, como formas, proporções e posições. Posteriormente, na ausência do objeto observado, pode-se reconstituí-lo na mente pela evocação de sua imagem memorizada $^{10}$. Como se percebe, o desenvolvimento do raciocínio espacial depende da memória espacial, que, por sua vez, depende da percepção espacial, tão facilitada com o uso de modelos tridimensionais, como os macromodelos odontológicos desenvolvidos para essa pesquisa.

Os consensos apresentados nas últimas questões e o campo destinados a comentários e sugestões comprovam balanço positivo do uso dos macromodelos tridimensionais como recurso complementar de ensino em escultura dentária. De forma geral, tal resultado veio ao encontro de todos os outros das questões anteriores: que a utilização dos macromodelos tridimensionais foi extrememamente válida para o processo do ensino e da aprendizagem dos alunos que foram submetidos à presente pesquisa.

\section{CONCLUSÕES}

De acordo com o objetivo da pesquisa, os estudantes entenderam que os macromodelos tridimensionais foram válidos como recurso facilitador da compreensão da técnica utilizada; levaram à melhora do processo de aprendizagem em comparação ao uso de slides; destacaram áreas de maior dificuldade no aprendizado; contribuíram para sedimentar os conceitos das formas geométricas das estruturas dentárias; e seriam por eles indicados para o aprendizado da disciplina. No entanto, não substituíram a demonstração prática dada pelo professor em sala de aula.

Esta é uma pesquisa preliminar sobre o tema proposto. Assim, há a necessidade de maior aprofundamento e discussão a respeito do tema, o que suscita a realização de novas pesquisas e estudos.

\section{ABSTRACT \\ 3D printed dental macromodels as a complementary resource for teaching dental}




\section{sculpture}

The diversification of pedagogical practices aimed at improving the teaching process. New methodologies, which favor the interests of students and to provide better understanding, can be important tools in the teaching and learning process. The aim of this study was to evaluate the perception of undergraduate dental students regarding the use of 3D-printed dental models as an additional resource for teaching dental sculpture at FACIPLAC. The sample consisted of 44 students enrolled in the fifth semester. A total of 44 sets were printed using four threedimensional models: maxillary central incisor, canine, first premolar and first molar, printed in plastic, resistant and colorful material. The sets were delivered to students at the beginning of each class and used to demonstrate the anatomical structures, collected at the end of the class, for improved memorization of knowledge. A questionnaire was applied regarding the use of the 3D models at the end of the school semester, and 38 answers were obtained. The research results indicated that the use of 3D models was a valid resource for facilitating technical understanding of dental sculpture; improved the learning process when compared to the use of slideshows; identified areas of greater difficulty in learning; contributed to the memorization of concepts and geometric shapes of tooth structures. Although students approved the use of 3D models, they did not replace practical demonstration.

Descriptors: Sculpture. Teaching. Learning. Printing, Three-Dimensional.

\section{REFERÊNCIAS}

1. Kaumaura D, Carvalho GL, Large-Marques JL, Antoniazzi JH. Avaliação do desempenho clínico dos alunos de graduação durante a prática da técnica endodôntica. Rev ABENO. 2003;3(1):33-40.

2. Perez FG. Linguagem total: uma pedagogia dos meios de comunicação. São Paulo: Sumus; 1978.

3. Haguenauer C. Metodologias e estratégias na educação à distância. Latec; 2005. [Acesso em
15 dez. 2017]. Disponível em: http://www. latec.ufrj.br/portfolio/at/4\%20EAD\%20metod ologias

4. Lana ESC. Impressora 3D [monografia]. Mogi das Cruzes: Universidade Braz Cubas; 2014.

5. Santos A. Tecnologias de informação e comunicação: limites e possibilidades no ensino superior. Anuário da produção acadêmica docente. 2011;5(12):129-50.

6. Kaczmarek H, Kwiatkowska I, Vukovi. Preparation and characterization of interpenetrating networks based on polyacrylates and poly (lactic acid). Express Polymer Letters. 2012;6(1):78-94.

7. Borges AB, Pucci CR, Torres CRG, Barcellos DC. Utilização de vídeo como recurso complementar de ensino em dentística operatória. Braz Dent Sci. 2009;12(3):6-10.

8. Blasca WQ, Maximino LP, Galdino DG, de Campos K, Picolini MM. Novas tecnologias educacionais no ensino da audiologia. Rev CEFAC. 2010;12(6):1017-24.

9. França EL, Pereira MB, Oliveira PF. O uso de modelos concretos e software no processo de ensino-aprendizagem de geometria molecular e arranjo espacial. XVI ENEQ/X EDUQUI; 2013.

10. Pertence AEM, Santos DMC, Jardim HV. Desenvolvimento de modelos didáticos para o ensino de desenho mecânico utilizando o conceito de prototipagem rápida. CABENGE. 2001. [Acesso em 15 dez. 2017]. Disponível em: http://cmundoinvisivel.blogspot.com/ 2010/12/desenvolvimento-de-modelosdidatic os.html

\section{Correspondência para:}

Andreia de Oliveira Souza

e-mail: aosdeia@gmail.com

SHCGN 705 Bloco F Casa 13 Asa Norte

70.730-766 Brasília/DF 